\title{
A FAMILY OF NUMBER FIELDS WITH UNIT RANK AT LEAST 4 THAT HAS EUCLIDEAN IDEALS
}

\author{
HESTER GRAVES AND M. RAM MURTY \\ (Communicated by Matthew A. Papanikolas)
}

\begin{abstract}
We will prove that if the unit rank of a number field with cyclic class group is large enough and if the Galois group of its Hilbert class field over $\mathbb{Q}$ is abelian, then every generator of its class group is a Euclidean ideal class. We use this to prove the existence of a non-principal Euclidean ideal class that is not norm-Euclidean by showing that $\mathbb{Q}(\sqrt{5}, \sqrt{21}, \sqrt{22})$ has such an ideal class.
\end{abstract}

\section{INTRODUCTION}

Euclidean ideals, introduced by Lenstra, generalize Euclidean algorithms in that the existence of a Euclidean algorithm for a domain $R$ implies that $R$ has trivial class group, while the existence of a Euclidean ideal in a domain $R$ implies that $R$ has cyclic class group. If an ideal is Euclidean, then so is every other ideal in its ideal class, and therefore we say the ideal class is Euclidean. If a domain $R$ has a Euclidean ideal class $[C]$, then $[C]$ generates the class group of $R$.

Lenstra showed (9), assuming the generalized Riemann hypothesis (henceforth abbreviated $\mathrm{GRH}$ ), that if $K$ is a number field with ring of integers $\mathcal{O}_{K}$ and class group $\mathrm{Cl}_{K}$, and if $\left|\mathcal{O}_{K}^{\times}\right|=\infty$, then

$$
\mathrm{Cl}_{K}=\langle[C]\rangle \text { if and only if }[C] \text { is a Euclidean ideal class. }
$$

Using techniques used by Harper and Murty (7], 8]), we will prove the following weaker result without assuming the GRH.

Theorem 1. Let $K$ be a number field, Galois over $\mathbb{Q}$, with ring of integers $\mathcal{O}_{K}$ and cyclic class group $\mathrm{Cl}_{K}$. If its Hilbert class field, $H(K)$, has an abelian Galois group over $\mathbb{Q}$ and if $\operatorname{rank}\left(\mathcal{O}_{K}^{\times}\right) \geq 4$, then

$$
\mathrm{Cl}_{K}=\langle[C]\rangle \text { if and only if }[C] \text { is a Euclidean ideal class. }
$$

\section{EUCLIDEAN IDEAL CLASSES}

The following is equivalent to Lenstra's definition [9] but is stated differently [4.

Definition 1. Suppose $R$ is a Dedekind domain and that $\mathbb{I}$ is the set of its non-zero integral ideals. If $C$ is an ideal of $R$, then it is called Euclidean if there exists a function $\psi: \mathbb{I} \longrightarrow W, W$ a well-ordered set, such that for all integral ideals $I$ and all $x \in I^{-1} C \backslash C$, there exists some $y \in C$ such that

$$
\psi\left((x-y) I C^{-1}\right)<\psi(I) .
$$

Received by the editors May 27, 2011 and, in revised form, October 5, 2011 and November 16, 2011.

2010 Mathematics Subject Classification. Primary 11-XX, 13F07.

(C) 2013 American Mathematical Society 2979 
We say $\psi$ is a Euclidean algorithm for $C$ and $C$ is a Euclidean ideal.

Generalizing the work of Malcolm Harper ([7]), the first author showed the following growth result ([3]).

Theorem 2. Let $K$ be a number field with ring of integers $\mathcal{O}_{K}^{\times}$and cyclic class group $\mathrm{Cl}_{K}$. Fix an ideal class $[C]$ in $\mathrm{Cl}_{K}$. If $\left|\mathcal{O}_{K}^{\times}\right|=\infty$ and if

$$
\left|\left\{\begin{array}{c}
\text { prime ideals } \\
\mathfrak{p} \subset \mathcal{O}_{K}
\end{array}: \mathrm{Nm}(\mathfrak{p}) \leq x,[\mathfrak{p}]=[C], \mathcal{O}_{K}^{\times} \rightarrow\left(\mathcal{O}_{K} / \mathfrak{p}\right)^{\times}\right\}\right| \gg \frac{x}{\log ^{2} x},
$$

then $[C]$ is a Euclidean ideal class.

\section{Primes AND Hilbert Class fields}

Suppose that $K$ is a number field Galois over $\mathbb{Q}$ and that its Hilbert class field $H(K)$ has abelian Galois group over $\mathbb{Q}$. Let $f(K)$ be the conductor of $K$, which is also the conductor of $H(K)$, so that both fields are contained in $\mathbb{Q}\left(\zeta_{f(K)}\right)$, where $\zeta_{f(K)}$ is a primitive $f(K)$-th root of unity. Note that $H(K)$ lies in $\mathbb{Q}\left(\zeta_{f(K)}\right)$ because $\operatorname{Gal}(H(K) / \mathbb{Q})$ is abelian. We define $d$ to be the smallest even number such that every root of unity in $K$ is a $d$-th root of unity.

Given a prime $p$ in $\mathbb{Q}$, we choose a prime $\mathfrak{p}$ in $K$ that lies above $p$, a prime $\mathfrak{P}$ in $H(K)$ that lies above $\mathfrak{p}$, and a prime $P$ in $\mathbb{Q}\left(\zeta_{f(K)}\right)$ that lies above $\mathfrak{P}$ :

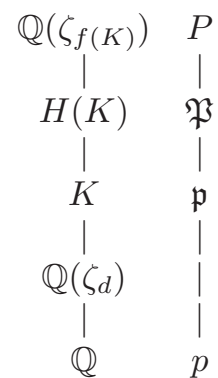

If $[C]$ generates the class group of $K$, then the Artin map maps all primes $\mathfrak{q}$ such that $[\mathfrak{q}]=[C]$ to a particular element $\sigma$ of $\mathrm{Gal}(H(K) / K)$ because $\mathrm{Cl}_{K} \cong$ $\operatorname{Gal}(H(K) / K)$. The Galois group $\operatorname{Gal}(H(K) / K)$ is isomorphic to

$$
\operatorname{Gal}\left(\mathbb{Q}\left(\zeta_{f(K)}\right) / K\right) / \operatorname{Gal}\left(\mathbb{Q}\left(\zeta_{f(K)}\right) / H(K)\right) .
$$

We can therefore identify $\operatorname{Gal}\left(H(K) / K\right.$ ) (and thus $\mathrm{Cl}_{K}$ ) with a set of elements in $\operatorname{Gal}\left(\mathbb{Q}\left(\zeta_{f(K)}\right) / \mathbb{Q}\right)$, as $\operatorname{Gal}\left(\mathbb{Q}\left(\zeta_{f(K)}\right) / K\right)$ is a subgroup of $\operatorname{Gal}\left(\mathbb{Q}\left(\zeta_{f(K)}\right) / \mathbb{Q}\right)$. By the isomorphism

$$
\tau: \operatorname{Gal}\left(\mathbb{Q}\left(\zeta_{f(K)}\right) / \mathbb{Q}\right) \rightarrow(\mathbb{Z} / f(K) \mathbb{Z})^{\times},
$$

we can see that there exists some $0<a<f(K),(a, f(K))=1$, such that if $p \equiv a$ $(\bmod f(K))$, then $\mathfrak{p}$ is of first degree and $[\mathfrak{p}]=[C]$.

This, along with Theorem 2, implies that in order to prove Theorem 1, it suffices to show that

$$
\mid\left\{\text { primes } p \leq x: p \equiv a \quad(\bmod f(K)), \mathcal{O}_{K}^{\times} \rightarrow\left(\mathcal{O}_{K} / \mathfrak{p}\right)^{\times}\right\} \mid \gg \frac{x}{\log ^{2} x},
$$

where the implied constant depends only on $K$. Using the linear sieve, we will show this when $\operatorname{rank}\left(\mathcal{O}_{K}^{\times}\right) \geq 4$. 


\section{The LINEAR SIEve}

The following notation is taken from Halberstam and Richert [6]. Suppose that $\mathfrak{A}$ is a finite set of integers, that $P$ is a collection of primes, and let $z \in \mathbb{R}, z \geq 2$. We define $S(\mathfrak{A} ; P, z)$ to be the number of elements of $\mathfrak{A}$ that are not divisible by any prime $p$ in $P$ such that $p \leq z$. It is a generalization of $\pi(y ; q, a)$, the number of primes less than or equal to $y$ which are congruent to $a(\bmod q)$. In order to bound $S(\mathfrak{A} ; P, z)$, we need to have a decent estimate for the size of $\mathfrak{A}$, which we denote by $X$.

For $q$ square-free, we define $\mathfrak{A}_{q}:=\{a \in \mathfrak{A}: a \equiv 0(\bmod q)\}$ and we choose a function $\omega_{0}$; we will be using $\frac{\omega_{0}(p)}{p} X$ to estimate $\left|\mathfrak{A}_{p}\right|$ for $p$ prime. The definition of $\omega_{0}$ is extended to all square-free $q$ by defining $\omega_{0}(1)=1$ and $\omega_{0}(q)=\prod_{p \mid q} \omega_{0}(p)$. In sieve theory we begin with this data, where we use approximations of the sizes of sets $\mathfrak{A}_{q}$ and keep track of the error terms that emanate from this calculation.

We now relate these definitions to the set of primes $P$. For ease of notation, we define the set of all primes not in $P$ to be $\bar{P}$, so that $P \cap \bar{P}=\emptyset$ and $P \cup \bar{P}$ is the set of all primes. For $p$ a prime, we define

$$
\omega(p)=\left\{\begin{array}{cl}
\omega_{0}(p) & \text { if } p \in P, \\
0 & \text { if } p \in \bar{P} .
\end{array}\right.
$$

For $q$ square-free, we define $\omega(1)=1, \omega(q)=\prod_{p \mid q} \omega(p)$, and

$$
R_{q}:=\left|\mathfrak{A}_{q}\right|-\frac{\omega(q)}{q} X \text { if } \mu(q) \neq 0,
$$

where $\mu$ is the Möbius function. The linear sieve can only be applied if the sets $\mathfrak{A}$ and $P$ satisfy certain conditions, enumerated below.

Condition 1. There exists a constant $A_{1} \geq 1$ such that

$$
0 \leq \frac{\omega(p)}{p} \leq 1-\frac{1}{A_{1}}
$$

Condition 2. There exist constants $L$ and $A_{2}$, both at least one, independent of $z$ and $w$, such that if $2 \leq w \leq z$, then

$$
-L \leq \sum_{w \leq p \leq z} \frac{\omega(p) \log p}{p}-\log \left(\frac{z}{w}\right) \leq A_{2} .
$$

In order to state Condition 3 , we define $(q, \bar{P})=1$ if every prime dividing $q$ is in $P$.

Condition 3. There exists an $\alpha, 0<\alpha \leq 1$, such that

$$
\sum_{\substack{q<\frac{X^{\alpha}}{(\log X)^{A_{4}}} \\(q, \bar{P})=1}} \mu^{2}(q) 3^{\nu(q)}\left|R_{q}\right| \leq A_{5} \frac{X}{\log ^{2} X} \quad(X \geq 2)
$$

for some constants $A_{4}, A_{5} \geq 1$. 
Theorem 3 (The linear sieve lower bound). If $\mathfrak{A}$ and $P$ satisfy Conditions 1,2, and 3 , and if $z \leq X$, then

$$
S(\mathfrak{A} ; P, z) \geq X \prod_{p<z}\left(1-\frac{\omega(p)}{p}\right)\left\{f\left(\alpha \frac{\log X}{\log z}\right)-B \frac{L}{(\log X)^{\frac{1}{14}}}\right\},
$$

where $B$ is an absolute constant and $f$ is a classical function defined in [6]. When $2 \leq u \leq 4, f(u):=\frac{2 e^{\gamma} \log (u-1)}{u}$, where $\gamma$ is the Euler-Mascheroni constant.

\section{Applying the linear sieve}

In order to prove that (1) holds in any number field $K$ satisfying the conditions in Theorem 1, we will show that for any given $x \in \mathbb{R}^{>0}, \mathfrak{A}, P$, and $z$ satisfy Conditions 1,2 , and 3 , where

$$
\mathfrak{A}=\left\{\frac{p-1}{d}: p \leq x, p \equiv a \quad(\bmod f(K)),\left(\frac{p-1}{d}, 2 f(K)\right)=1\right\}
$$

and $P$ is the set of primes $\leq z$. The $a$ in the definition of $\mathfrak{A}$ is chosen so that if $p \equiv a$ $(\bmod f(K))$, then $[\mathfrak{p}]=[C]$ and $\mathfrak{p}$ is of degree one. Note that $p \equiv 1(\bmod d)$. Since

$$
\left|\left\{\frac{p-1}{d}: p \leq x, p \equiv a \quad(\bmod f(K))\right\}\right| \sim \frac{\operatorname{li}(x)}{\phi(f(K))},
$$

$|\mathfrak{A}| \sim \frac{C \mathrm{li}(x)}{\phi(f(K))}$ by the Eratosthenes sieve for some constant $0<C<1$ that depends only on $f(K)$.

If $p^{\prime}$ is a prime, $p^{\prime} \leq x$, and $p^{\prime} \nmid 2 f(K)$, then $\left|\mathfrak{A}_{p^{\prime}}\right| \sim \frac{C \operatorname{li}(x)}{\phi(f(K)) \phi\left(p^{\prime}\right)}$, so

$$
\frac{\omega_{0}\left(p^{\prime}\right)}{p^{\prime}}=\frac{1}{\phi\left(p^{\prime}\right)}
$$

If $p^{\prime}$ is a prime, $p^{\prime} \leq x$, and $p^{\prime} \mid 2 f(K)$, then $\left|\mathfrak{A}_{p^{\prime}}\right|=0$, so $\frac{\omega_{0}\left(p^{\prime}\right)}{p^{\prime}}=0$. From this, we see that for all square-free $q$,

$$
\omega(q)=\left\{\begin{array}{cc}
\frac{q}{\phi(q)} & \text { if }(q, \bar{P})=1,(q, 2 f(K))=1, \\
0 & \text { otherwise. }
\end{array}\right.
$$

Lemma 1. The sets $\mathfrak{A}$ and P satisfy Condition 1.

Proof. Note that for $p>2, \frac{\omega(p)}{p}=\frac{1}{\phi(p)}$ or 0 and

$$
0 \leq \frac{1}{\phi(p)} \leq \frac{1}{2}=1-\frac{1}{2}
$$

As $2 \mid 2 f(K)$, we can see that $0 \leq \frac{\omega(2)}{2}=0<1-\frac{1}{2}$.

Lemma 2. The set $\mathfrak{A}$, the set $P$, and the quantity $z$ satisfy Condition 2.

Proof. Suppose that $2 \leq w \leq z$. Then

$$
\sum_{w \leq p \leq z} \frac{\omega(p) \log p}{p}=\sum_{w \leq p \leq z} \frac{\frac{p}{p-1} \log p}{p}-\sum_{w \leq p \leq z, p \mid 2 f(K)} \frac{\frac{p}{p-1} \log p}{p}
$$


and as

we know that

$$
\sum_{w \leq p \leq z, p \mid 2 f(K)} \frac{\frac{p}{p-1} \log p}{p} \leq \sum_{p \mid 2 f(K)} \frac{\frac{p}{p-1} \log p}{p}=\mathcal{O}(1),
$$

(2) $\sum_{w \leq p \leq z} \frac{\omega(p) \log p}{p}-\log \left(\frac{z}{w}\right)=\sum_{w \leq p \leq z} \frac{\log p}{p}+\sum_{w \leq p \leq z} \frac{\log p}{p(p-1)}-\log \left(\frac{z}{w}\right)-\mathcal{O}(1)$.

The sequence $\sum_{p \leq x} \frac{\log p}{p(p-1)}=\mathcal{O}(1)$. By Chebyshev's Theorem (see [1], p. 6),

$$
\sum_{p \leq x} \frac{\log p}{p}=\log x+\mathcal{O}(1)
$$

so (2) becomes

$$
\log z-\log w-\log \left(\frac{z}{w}\right)+\mathcal{O}(1)=\mathcal{O}(1)
$$

To prove that Condition 3 holds, we must first prove the following lemma.

Lemma 3. If $c \in \mathbb{N}$, then

$$
\sum_{\substack{(q, \bar{P})=1 \\ q \leq z \\ q \text { square-free }}} \frac{c^{\nu(q)}}{q} \ll \log ^{c} z,
$$

where $\nu(q)$ is the number of distinct prime factors of $q$.

Proof. Note that

$$
\sum_{\substack{(q, \bar{P})=1 \\ q \leq z \\ q \text { square-free }}} \frac{c^{\nu(q)}}{q} \leq \prod_{p \leq z}\left(1+\frac{c}{p}\right) \leq \prod_{p \leq z}\left(1+\frac{1}{p}\right)^{c} .
$$

Now

$$
\prod_{p \leq z}\left(1+\frac{1}{p}\right) \leq \prod_{p \leq z}\left(\sum_{j=0}^{\infty} \frac{1}{p^{j}}\right)=\prod_{p \leq z}\left(1-\frac{1}{p}\right)^{-1} .
$$

Mertens' Theorem (see [10], p. 128) states that

$$
\prod_{p \leq z}\left(1-\frac{1}{p}\right)=\frac{e^{-\gamma}}{\log z}\left(1+\mathcal{O}\left(\frac{1}{\log z}\right)\right)
$$

where $\gamma$ is the Euler constant. Thus

$$
\prod_{p \leq z}\left(1-\frac{1}{p}\right)^{-1}=e^{\gamma} \log z\left(1+\mathcal{O}\left(\frac{1}{\log z}\right)\right)^{-1} .
$$

Noting that

we see

$$
\left(1+\mathcal{O}\left(\frac{1}{\log z}\right)\right)^{-1}=1+\mathcal{O}\left(\frac{1}{\log z}\right),
$$

$$
\prod_{p \leq z}\left(1-\frac{1}{p}\right)^{-1}=e^{\gamma} \log z+\mathcal{O}(1) .
$$


Thus

$$
\prod_{p \leq z}\left(1+\frac{1}{p}\right)^{c}=\mathcal{O}\left(\log ^{c}(z)\right)
$$

Lemma 4. The sets $\mathfrak{A}$ and P satisfy Condition 3.

Proof. According to the Bombieri-Vinogradov inequality (see [1, p. 39), there exists some $B>1$ such that

$$
\sum_{q \leq \frac{x^{\frac{1}{2}}}{\log B-1} x} \max _{y \leq x} \max _{(\alpha, q)=1}\left|\pi(y ; q, \alpha)-\frac{\operatorname{li}(y)}{\phi(q)}\right| \ll \frac{x}{\log ^{13} x} .
$$

By applying Cauchy-Schwarz (see [1, p. 27), we see that

$$
\begin{aligned}
& \sum_{\substack{q \leq \frac{1}{2} \\
q \leq \frac{x^{2}}{\log ^{B} x} \\
(q, \bar{P})=1}} \mu^{2}(q) 3^{\nu(q)}\left|R_{q}\right| \leq \sum_{\substack{q \leq \frac{x^{\frac{1}{2}}}{q} \\
(q, \bar{P})^{B}=1}} 3^{\nu(q)}\left|R_{q}\right| \\
& \leq \sqrt{\sum_{\substack{q \leq \frac{x^{\frac{1}{2}}}{\left(q, \log ^{B} x\right.} \\
(q, \bar{P})=1}} 9^{\nu(q)\left|R_{q}\right|}} \sqrt{\sum_{\substack{q \leq \frac{x^{2}}{2} \\
(q, \bar{P})=1}}\left|R_{q}\right| .}
\end{aligned}
$$

Let us examine the first term in the product. We can see that for $q$ square-free,

$$
\left|R_{q}\right| \sim\left(\left|\mathfrak{A}_{q}\right|-\frac{C \operatorname{li}(x)}{\phi(q f(K))}\right) \text { if }(q, \bar{P})=(q, 2 f(K))=1
$$

and

$$
\left|R_{q}\right|=0 \text { otherwise, }
$$

so

$$
\left|R_{q}\right| \leq\left|\mathfrak{A}_{q}\right|+\left|\frac{C \operatorname{li}(x)}{\phi(q f(K))}\right| \leq \frac{2 x}{q} .
$$

Therefore

$$
\sum_{\substack{q \leq \frac{x^{\frac{1}{2}}}{q} \log ^{B} x \\(q, \bar{P})=1}} 9^{\nu(q)}\left|R_{q}\right| \leq 2 x \sum_{\substack{q \leq \frac{x^{\frac{1}{2}}}{q} \log ^{B} x \\(q, \bar{P})=1 \\ q \text { square-free }}} \frac{9^{\nu(q)}}{q} \leq 2 x \sum_{\substack{q \leq \frac{x^{\frac{1}{2}}}{\left(\log ^{B} x\right.} \\(q, \bar{P})=1 \\ q \text { square-free }}} \frac{9^{\nu(q)}}{q} \ll 2 x \log ^{9} x
$$

by Lemma 3 . 
We shall now examine the second term in the product. By definition, if $(q, 2 f(K))$ $\neq 1$, then $\left|R_{q}\right|=0$. If $(q, 2 f(K))=1=(q, \bar{P})$, then

$$
\begin{aligned}
& \left|R_{q}\right|=|| \mathfrak{A}_{q}\left|-\frac{1}{\phi(q)}\right| \mathfrak{A}|| \\
& =\left|\sum_{\substack{p \leq x \\
p \equiv a \bmod (f(K)) \\
p=1 \bmod (q) \\
\left(\frac{p-1}{d}, 2 f(K)\right)=1}} 1-\frac{1}{\phi(q)} \sum_{\substack{p \leq x \\
p=a \bmod (f(K)) \\
\left(\frac{p-1}{d}, 2 f(K)\right)=1}} 1\right| \\
& =\left|\sum_{\substack{p \leq x \\
p \equiv a \bmod (f(K)) \\
p \equiv 1 \bmod (q)}} \sum_{l \mid\left(\frac{p-1}{d}, 2 f(K)\right)} \mu(l)-\frac{1}{\phi(q)} \sum_{\substack{p \leq x \\
p \equiv a \bmod (f(K))}} \sum_{l \mid\left(\frac{p-1}{d}, 2 f(K)\right)} \mu(l)\right| \\
& =\left|\sum_{l \mid 2 f(K)} \mu(l)\left(\sum_{\substack{p \leq x \\
p \equiv a \bmod (f(K)) \\
p \equiv 1 \bmod (q) \\
p \equiv 1 \bmod (d l)}} 1-\frac{1}{\phi(q)} \sum_{\substack{p \leq x \\
p \equiv a \bmod (f(K)) \\
p \equiv 1 \bmod (d l)}} 1\right)\right| \\
& \leq \sum_{l \mid 2 f(K)}\left|\pi\left(x, q[f(K), d l], a^{\prime}\right)-\frac{1}{\phi(q)} \pi\left(x,[f(K), d l], a^{*}\right)\right|,
\end{aligned}
$$

where $a^{\prime}$ is the smallest positive solution to $a^{\prime} \equiv a(\bmod f(K)), a^{\prime} \equiv 1(\bmod q), a^{\prime}$ $\equiv 1(\bmod d l) ;$ where $a^{*}$ is the smallest positive solution to $a^{*} \equiv a(\bmod f(K)), a^{*} \equiv$ $1(\bmod d l) ;$ and where $\left[c_{1}, \cdots, c_{k}\right]=\operatorname{lcm}\left(c_{1}, \cdots, c_{k}\right)$. This means that

$$
\begin{aligned}
& \sum_{\substack{q \leq \frac{x^{\frac{1}{2}}}{q \log ^{B} x} \\
(q, \bar{P})=1}}\left|R_{q}\right| \leq \sum_{\substack{q \leq \frac{x^{\frac{1}{2}}}{\left(q, \overline{\log ^{B} x}\right.} \\
(q)=1}}\left|\pi\left(x, q[f(K), d l], a^{\prime}\right)-\frac{\operatorname{li}(x)}{\phi(q[f(K), d l])}\right| \\
& +\sum_{\substack{q \leq \frac{x^{\frac{1}{2}}}{\left(\log ^{B} x\right.} \\
(q, \bar{P})=1}}\left|\frac{\operatorname{li}(x)}{\phi(q[f(K), d l])}-\frac{1}{\phi(q)} \pi\left(x,[f(K), d l], a^{*}\right)\right| .
\end{aligned}
$$

Note that

$$
\begin{aligned}
& \sum_{\substack{q \leq \frac{x^{\frac{1}{2}}}{\left(q, \log ^{B} x\right.} \\
(q, P)=1}}\left|\pi\left(x, q[f(K), d l], a^{\prime}\right)-\frac{\operatorname{li}(x)}{\phi(q[f(K), d l])}\right| \\
& \leq \sum_{q \leq \frac{[f(K), d l] x}{\frac{1}{2}}}\left|\pi\left(x, q, a^{\prime}\right)-\frac{\operatorname{li}(x)}{\phi(q)}\right| \leq \sum_{q \leq \frac{[f(K), d] x_{x}^{\frac{1}{2}}}{\log ^{B} x}} \max _{(r, q)=1}\left|\pi(x, q, r)-\frac{\operatorname{li}(x)}{\phi(q)}\right| .
\end{aligned}
$$


By definition, as $(q,[f(K), d l])=1$,

$$
\frac{1}{\phi(q)} \pi\left(x,[f(K), d l], a^{*}\right)=\frac{1}{\phi(q)} \sum_{r=0}^{q-1} \pi\left(x, q[f(K), d l], a_{r}^{*}\right),
$$

where $a_{r}^{*}$ is the smallest positive solution to

$$
t \equiv a^{*} \quad(\bmod [f(K), d l]), t \equiv r \quad(\bmod q)
$$

The sum $\sum_{(r, q) \neq 1} \pi\left(x,[f(K), d l], a_{r}^{*}\right)$ counts all the primes less than or equal to $x$ that are equivalent to $a^{*}(\bmod [f(K), d l])$ and $r(\bmod q)$, where $r$ is not relatively prime to $q$. If $p \equiv r(\bmod q)$ and $(r, q) \neq 1$, then $p$ must divide $q$. Our sum, therefore, is bounded above by $\sum_{p \mid q} 1=\nu(q) \leq \frac{\log q}{\log 2}$, so

$$
\begin{gathered}
\frac{1}{\phi(q)} \sum_{r=0}^{q-1} \pi\left(x, q[f(K), d l], a_{r}^{*}\right)=\frac{1}{\phi(q)} \nu(q)+\frac{1}{\phi(q)} \sum_{(r, q)=1} \pi\left(x, q[f(K), d l], a_{r}^{*}\right) \\
=\frac{1}{\phi(q)} \sum_{(r, q)=1} \pi(x, q[f(K), d l], r)+\frac{1}{\phi(q)} \nu(q) .
\end{gathered}
$$

This is used to rewrite

$$
\sum_{\substack{q \leq \frac{1}{2} \\\left(q, \log ^{B} x\right.}}\left|\frac{\operatorname{li}(x)}{\phi(q[f(K), d l])}-\frac{1}{\phi(q)} \pi\left(x,[f(K), d l], a^{*}\right)\right|
$$

as

$$
\sum_{\substack{q \leq \frac{x^{\frac{1}{2}}}{q \log ^{B} x} \\(q, \bar{P})=1}}\left|\frac{\nu(q)}{\phi(q)}+\frac{1}{\phi(q)} \sum_{(r, q)=1} \pi(x, q[f(K), d l], r)-\frac{\operatorname{li}(x)}{\phi(q[f(K), d l])}\right|,
$$

which is bounded above by

$$
\begin{aligned}
& \sum_{\substack{q \leq \frac{x^{\frac{1}{2}}}{\log ^{B} x} \\
(q, \bar{P})=1}} \frac{\nu(q)}{\phi(q)}+\left|\sum_{(r, q)=1} \frac{\pi(x, q[f(K), d l], r)}{\phi(q)}-\frac{\operatorname{li}(x)}{\phi(q) \phi(q[f(K), d l])}\right| \\
& \leq \sum_{\substack{q \leq \frac{x^{\frac{1}{2}}}{\log ^{B} x}\\
}} \frac{\log q}{\log 2}+\max _{(r, q)=1}\left|\pi(x, q[f(K), d l], r)-\frac{\operatorname{li}(x)}{\phi(q[f(K), d l])}\right| .
\end{aligned}
$$


Putting the pieces together, we see that

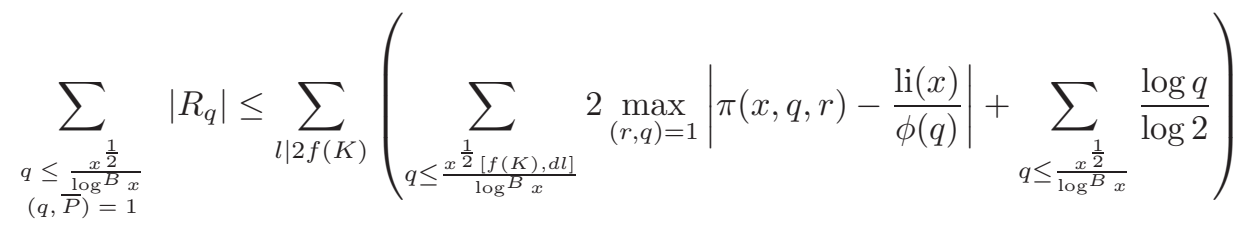

$$
\begin{aligned}
& \ll \frac{x^{\frac{1}{2}}}{\log ^{B-1} x}+\sum_{q \leq \frac{x^{\frac{1}{2}}[f(K), d l]}{\log ^{B} x}} \max _{(r, q)=1}\left|\pi(x, q, r)-\frac{\operatorname{li}(x)}{\phi(q)}\right| \\
& \ll \frac{x^{\frac{1}{2}}}{\log ^{B-1} x}+\sum_{q \leq \frac{x^{\frac{1}{2}}}{\log ^{B-1} x}} \max _{(r, q)=1}\left|\pi(x, q, r)-\frac{\operatorname{li}(x)}{\phi(q)}\right| \\
& \ll \frac{x^{\frac{1}{2}}}{\log ^{B-1} x}+\frac{x}{\log ^{13} x} \ll \frac{x}{\log ^{13} x} .
\end{aligned}
$$

In conclusion,

$$
\sum_{\substack{q \leq \frac{x^{\frac{1}{2}}}{q} \\\left(q, \overline{\log ^{B} x}\right.}} \mu^{2}(q) 3^{\nu(q)}\left|R_{q}\right| \ll \sqrt{2 x \log ^{9} x} \sqrt{\frac{x}{\log ^{13} x}} \ll \frac{x}{\log ^{2} x} .
$$

We may now apply the linear sieve to $\mathfrak{A}$.

Lemma 5. For any small $\epsilon>0$, there exists a positive constant $k$ such that if $x$ is large enough, then

$$
S\left(\mathfrak{A}, P, x^{\frac{1-\epsilon}{4}}\right) \geq k \frac{x}{\log ^{2} x} .
$$

Proof. By Lemmas 1, 2, and 4 we may apply the linear sieve to $\mathfrak{A}$, implying that

$$
\begin{aligned}
& S\left(\mathfrak{A}, P, x^{\frac{1-\epsilon}{4}}\right) \\
& \geq \frac{C \operatorname{li}(x)}{\phi(f(K))} \prod_{\substack{p<x \\
p<\frac{1-\epsilon}{4} \\
(p, 2 f(K))=1}}\left(1-\frac{1}{\phi(p)}\right)\left\{f\left(\frac{1}{2} \frac{\log \left(\frac{C x}{\phi(f(K)) \log x}\right)}{\log x^{\frac{1-\epsilon}{4}}}\right)-\frac{B L}{(\log x)^{\frac{1}{14}}}\right\} \\
& \gg \frac{C x}{\phi(f(K)) \log x} \prod_{\substack{p<x^{\frac{1-\epsilon}{4}} \\
(p, 2 f(K))=1}}\left(1-\frac{1}{\phi(p)}\right) \\
& \quad \times\left\{f\left(\frac{1}{2} \frac{\log \left(\frac{C x}{\phi(f(K)) \log x}\right)}{\log x^{\frac{1-\epsilon}{4}}}\right)-\frac{B L}{(\log x)^{\frac{1}{14}}}\right\} .
\end{aligned}
$$


For $0<\epsilon^{\prime}<\epsilon$ and for $x$ large enough,

$$
\begin{aligned}
& \frac{1}{2}\left(\frac{\log x-\log \log x+\log \left(\frac{C}{\phi(f(K))}\right)}{\left(\frac{1-\epsilon}{4}\right) \log x}\right) \leq \frac{1}{2}\left(\frac{\log x}{\left(\frac{1-\epsilon}{4}\right) \log x}\right)=\frac{2}{1-\epsilon}<4 \text { and } \\
& \frac{1}{2}\left(\frac{\log x-\log \log x+\log \left(\frac{C}{\phi(f(K))}\right)}{\left(\frac{1-\epsilon}{4}\right) \log x}\right) \geq \frac{1}{2}\left(\frac{\log x\left(1-\epsilon^{\prime}\right)}{\left(\frac{1-\epsilon}{4}\right) \log x}\right)=\frac{2\left(1-\epsilon^{\prime}\right)}{1-\epsilon}>2,
\end{aligned}
$$

so that $f\left(\frac{1}{2} \frac{\log \left(\frac{C x}{\phi(f(K) \log x}\right)}{\log x^{\frac{1-\epsilon}{4}}}\right)$ is bounded below by a positive constant.

Thus there exist constants $C_{1}, C_{2}>0$ such that for large enough $x$,

$$
\begin{aligned}
& S\left(\mathfrak{A}, P, x^{\frac{1-\epsilon}{4}}\right) \geq\left(C_{1} \frac{x}{\log x}-C_{2} \frac{x}{(\log x)^{\frac{15}{14}}}\right) \prod_{p \leq x^{\frac{1-\epsilon}{4}}}\left(1-\frac{1}{p}\right) \\
\geq & \left(C_{1} \frac{x}{\log x}-C_{2} \frac{x}{(\log x)^{\frac{15}{14}}}\right) \frac{e^{-\gamma}}{\log \left(x^{\frac{1-\epsilon}{4}}\right)}\left(1+\mathcal{O}\left(\frac{1}{\log \left(x^{\frac{1-\epsilon}{4}}\right)}\right)\right)
\end{aligned}
$$

by Mertens' Theorem (see [10, p. 128), and so there exists some $k>0$ such that for large enough $x$,

$$
S\left(\mathfrak{A}, P, x^{\frac{1-\epsilon}{4}}\right) \geq k \frac{x}{\log ^{2} x} .
$$

\section{Proof of Theorem 1}

In order to prove Theorem [1, we first need to state the following definition and the Gupta-Murty bound [5].

Definition 2. If $\mathfrak{M}$ is a monoid in $\mathcal{O}_{K}$ such that its elements are relatively prime to an ideal $I$, then we define $f_{\mathfrak{M}}(I)$ to be the size of the image of $\mathfrak{M}$ in $\left(\mathcal{O}_{K} / \mathfrak{p}\right)^{\times}$. We define $f(I)$ to be $f_{\mathcal{O}_{K}^{\times}}(I)$.

Proposition 1 (The Gupta-Murty bound [5]). If $\mathfrak{M}$ is a monoid in $\mathcal{O}_{K}^{\times}$containing $t$ multiplicatively independent elements, then

$$
\left|\left\{\mathfrak{p}: f_{\mathfrak{M}}(\mathfrak{p}) \leq x\right\}\right| \ll x^{\frac{t+1}{t}} .
$$

We can now prove Theorem 1

Proof of Theorem 1. Recall that, by Lemma 5.

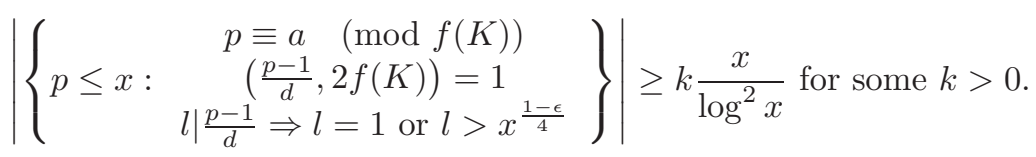

For the following, suppose that $p$ is one of the primes in the above set and that $\mathfrak{p}$ lies above it in $K$. Since $p \equiv a(\bmod f(K)), \operatorname{Nm}(\mathfrak{p})=p$ and $f(\mathfrak{p}) \mid(p-1)$. Note that $\mathcal{O}_{K}^{\times} \rightarrow\left(\mathcal{O}_{K}^{\times} / \mathfrak{p}\right)^{\times}$if and only if $f(\mathfrak{p})=p-1$.

As $d \nmid f(\mathfrak{p})$ if and only if $p \mid \operatorname{Nm}\left(1-\zeta_{d}^{r-1}\right)$ for some $r$ such that $(r, d)=1, d \nmid f(\mathfrak{p})$ implies that $p \mid d$. This is a contraction as $p \equiv a \equiv 1(\bmod d)$, so $d \mid f(\mathfrak{p})$, and we can see that if $l \mid \frac{p-1}{f(\mathfrak{p})}$, then $l=1$ or $l>x^{\frac{1-\epsilon}{4}}$. Thus either $\frac{p-1}{f(\mathfrak{p})}=1$ or $\frac{p-1}{f(\mathfrak{p})}>x^{\frac{1-\epsilon}{4}}$. 
If $\frac{p-1}{f(\mathfrak{p})} \neq 1$, we can see that $x^{\frac{3+\epsilon}{4}}>f(\mathfrak{p})$. The Gupta-Murty bound implies that $\left|\left\{\mathfrak{p}: f(\mathfrak{p}) \leq x^{\frac{3+\epsilon}{4}}\right\}\right| \ll x^{\frac{3+\epsilon}{4} \frac{5}{4}}=x^{\frac{15+5 \epsilon}{16}}$ since we assumed that $\operatorname{rank}\left(\mathcal{O}_{K}^{\times}\right) \geq 4$.

This implies that

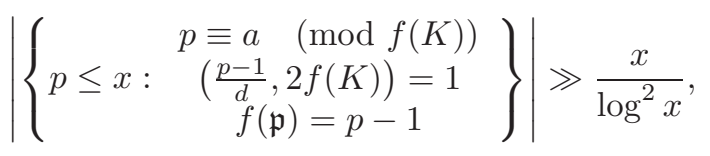

so

$$
\left|\left\{p \leq x: \begin{array}{c}
{[\mathfrak{p}]=[C]} \\
\mathcal{O}_{K}^{\times} \rightarrow\left(\mathcal{O}_{K} / \mathfrak{p}\right)^{\times}
\end{array}\right\}\right| \gg \frac{x}{\log ^{2} x},
$$

and thus $[C]$ is a Euclidean ideal class by Theorem 2 .

\section{Application}

When Lenstra defined Euclidean ideals, he was initially inspired by rings for which the algebraic norm of its elements is a Euclidean algorithm, leading him to define norm-Euclidean ideals [9].

Definition 3. If $K$ is a number field and $C$ is a fractional ideal of $\mathcal{O}_{K}$, then $C$ is norm-Euclidean if for all $x \in K$, there exists some $y \in C$ such that

$$
\operatorname{Nm}(x-y)<\operatorname{Nm}(C) .
$$

One can check that this is equivalent to $\psi=\mathrm{Nm}$ in Definition 1. If $C$ is normEuclidean, then we say that $[C]$ is a norm-Euclidean ideal class. A ring can have at most one norm-Euclidean ideal class [9].

In the same paper, Lenstra showed that $K$ has a non-principal Euclidean ideal if $K=\mathbb{Q}(\sqrt{d})$, for $d=-20,-15,40,60$ and $85[9$. In each of these situations, the class number is two and the generating ideal is norm-Euclidean. These examples were found without assuming GRH [9], 2]. The only other example in the literature that does not assume $\mathrm{GRH}$ is $\mathbb{Q}(\sqrt{2}, \sqrt{35})$, which has class number two [4. It is unknown whether the generating ideal is norm-Euclidean.

Proposition 2. The field $\mathbb{Q}(\sqrt{5}, \sqrt{21}, \sqrt{22})$ has a non-principal Euclidean ideal class that is not norm-Euclidean.

Proof. If one enters the commands

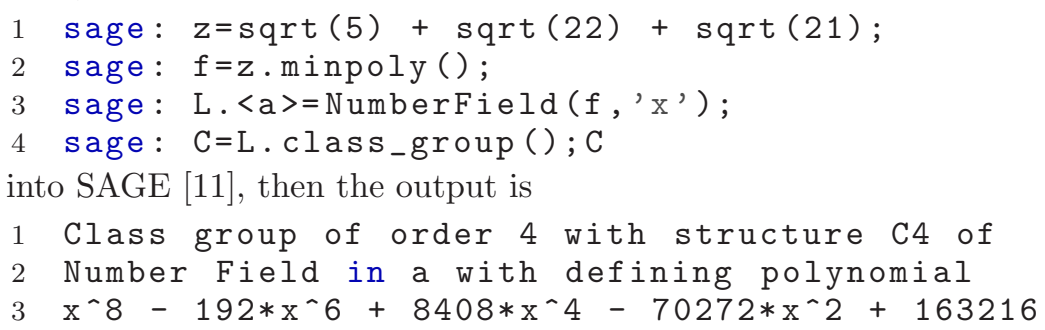

Thus the class group of $\mathbb{Q}(\sqrt{5}, \sqrt{21}, \sqrt{22})$ is cyclic and of size four. The field $\mathbb{Q}(\sqrt{2}, \sqrt{3}, \sqrt{5}, \sqrt{7}, \sqrt{11})$ is an unramified, degree four extension of $\mathbb{Q}(\sqrt{5}, \sqrt{21}, \sqrt{22})$ and is therefore its Hilbert class field. 
As

$$
\operatorname{Gal}(\mathbb{Q}(\sqrt{2}, \sqrt{3}, \sqrt{5}, \sqrt{7}, \sqrt{11}) / \mathbb{Q}) \cong(\mathbb{Z} / 2 \mathbb{Z})^{5}
$$

and $\operatorname{rank}\left(\mathcal{O}_{\mathbb{Q}(\sqrt{5}, \sqrt{21}, \sqrt{22})}\right)=7>4$, both generators of the class group of $\mathbb{Q}(\sqrt{5}, \sqrt{21}$, $\sqrt{22}$ ) are Euclidean ideal classes by Theorem 1. At most one generator can be normEuclidean, so $\mathbb{Q}(\sqrt{5}, \sqrt{21}, \sqrt{22})$ has a non-principal Euclidean ideal class that is not norm-Euclidean.

\section{REFERENCES}

[1] Alina Carmen Cojocaru and M. Ram Murty, An Introduction to Sieve Methods and Their Applications, London Mathematical Society Student Texts, 66. Cambridge University Press, Cambridge, 2006. MR2200366 (2006k:11184)

[2] Hester Graves and Nick Ramsey, Euclidean Ideals in Quadratic Imaginary Fields, Journal of the Ramanujan Math Society, 26, no. 1, March 2011. MR2789745

[3] Hester Graves, Growth Results and Euclidean Ideals, submitted, arXiv:1008.2479.

[4] Hester Graves, $\mathbb{Q}(\sqrt{2}, \sqrt{35})$ has a non-principal Euclidean ideal, Int. J. Number Theory 7 , no. 8, 2269-2271, 2011. MR2873154

[5] Rajiv Gupta and M. Ram Murty, A remark on Artin's conjecture, Invent. Math. 78, 127-130, 1984. MR762358 (86d:11003)

[6] H. Halberstam and H.-E. Richert, Sieve Methods, Academic Press, New York, 1974. MR 0424730(54:12689)

[7] M. Harper, $\mathbb{Z}[\sqrt{14}]$ is Euclidean, Canad. J. Math. 56, 55-70, 2004. MR2031122|(2005f:11236)

[8] M. Harper and M. Ram Murty, Euclidean rings of algebraic integers, Canad. J. Math. 56, 71-76, 2004. MR2031123 (2005h:11261)

[9] H.K. Lenstra, Euclidean ideal classes, Astérisque 61, 121-131, 1979. MR556669(81b:12005)

[10] M. Ram Murty, Problems in Analytic Number Theory, GTM, 206, Springer, New York, 2001. MR:1803093(2001k:11002)

[11] William Stein, SAGE Mathematics Software (version 4.4.4), The SAGE Group, 2010, http://www.sagemath.org/.

Department of Mathematics, Queen's University, 99 University Avenue, Kingston, ONTARIO, K7L 3N6, CANADA

E-mail address: gravesh@mast.queensu.ca

Department of Mathematics, Queen's University, 99 University Avenue, Kingston, Ontario, K7L 3N6, CANADA 